\title{
Articulação do serviço social hospitalar com a rede de apoio informal em cuidados continuados
}

\section{Mediation between hospital social work and the informal support network in continuing healthcare}

\author{
Paula Macedo (1) \\ Sónia Guadalupe $(2,3)$ \\ (1) Hospital Distrital de Pombal do Centro Hospitalar de Leiria, Portugal \\ (2) Instituto Superior Miguel Torga, Centro de Investigação Interdisciplinar Psicossocial, Portugal \\ (3) Centro de Estudos e Investigação em Saúde da Universidade de Coimbra, Portugal
}

Recebido: 03/11/2020; Revisto: 16/11/2020; Aceite: 21/11/2020.

https://doi.org/10.31211/rpics.2020.6.2.189

D) Check for updates

\begin{abstract}
Resumo
Objetivo: O estudo teve como objetivo analisar a articulação entre a saúde e o apoio social informal no âmbito da continuidade de cuidados. Participantes: Participaram 57 doentes referenciados em 2019 por um hospital para a Rede Nacional de Cuidados Continuados Integrados em Portugal. A amostra era maioritariamente do sexo feminino $(63,2 \% ; n=36)$, com uma média de idades de 80 anos $(D P=9,2)$, predominantemente de residência rural $(64,9 \% ; n=37)$, casada $(56,1 \% ; n=32)$, em que $35,0 \%$ vivia só $(n=20)$. Método: Estudo descritivo com análise quantitativa univariada e análise categorial do conteúdo dos registos no processo social. Resultados: Dos 57 doentes referenciados para a Rede, registaram-se óbitos e cancelamentos em 32 casos $(56,1 \%)$. Dos 25 doentes que tiveram alta da Rede, no pós-alta todos necessitavam de apoio informal e/ou formal, enquanto que à data de referenciação hospitalar apenas $20,0 \%$ da amostra necessitava de apoio $(p<0,001)$. Dos que tinham apoio, cerca de um terço tinha apoio da rede social primária, maioritariamente dos filhos e cerca de metade dos doentes tinha filhos emigrantes. No pós-alta $64,0 \%$ ( $n=16$ dos 25 ) tinham apoio informal. Verificámos ainda que 8,8\% dos doentes eram cuidadores e passaram a necessitar de cuidados. Conclusão: $O$ estudo confirma a importância de assegurar cuidados continuados aos cidadãos que viram a sua vulnerabilidade aumentada por doença com sequelas, assim como a fulcral articulação com o sistema de apoio informal aos doentes.
\end{abstract}

Palavras-Chave: Cuidados Continuados; Serviço Social; Saúde; Envelhecimento; Apoio social informal.

\section{DI\&D | ISMT}

rpics@ismt.pt

https://rpics.ismt.pt
Publicação em Acesso Aberto

(c) 2020. O(s) Autor(es). Este é um artigo de acesso aberto distribuído sob a Licença Creative Commons Attribution, que permite uso, distribuição e reprodução sem restrições em qualquer meio, desde que o trabalho original seja devidamente citado.

\author{
Paula Macedo \\ Hospital Distrital de Pombal do Centro \\ Hospitalar de Leiria, Leiria, Portugal \\ E-mail: pmariamacedo@gmail.com
}




\begin{abstract}
Objective: The study aimed to analyze the mediation between healthcare and informal social support in the continuing healthcare process. Participants: fifty-seven patients were referred in 2019 by a hospital to the Rede Nacional de Cuidados Continuados Integrados in Portugal (a continuing healthcare services network). The sample was mostly female $(63.2 \% ; n=36)$, with an average age of 80 years old $(D P=9.2)$, predominantly from rural areas $(64.9 \% ; n=37)$, married $(56.1 \% ; n=32)$, and $35.0 \%$ lived alone ( $n$ $=20$ ). Methods: Descriptive study with univariate quantitative analysis and categorical content analysis of social work data. Results: Of the 57 patients referred to the Network, deaths and cancellations were registered in 32 cases (56.1\%). Of the 25 patients discharged from the Network in the post-discharge period, all needed informal and/or formal support, while at the hospital referral date, only $20.0 \%$ of the sample needed support $(p<.001)$. Of those who had support, about a third had support from the primary social network, mostly from their sons or daughters, and about half of the patients had emigrant children. At post-discharge $64.0 \%$ ( $n=16$ out of 25 ) had informal support. We also found that $8.8 \%$ of the patients were caregivers and began to require care. Conclusion: The study confirms the importance of ensuring continued care for citizens who have seen their vulnerability increased by illness, as well as the centrality of the informal support system for patients.
\end{abstract}

Keywords: Continuing healthcare; Social work; Health; Aging; Informal social support.

\title{
Introdução
}

A esfera dos cuidados informais sofre uma pressão crescente na relação com os sistemas de saúde. As alterações demográficas, com o aumento tendencial da população idosa e do envelhecimento populacional, a crescente prevalência de doenças crónicas, o aumento da morbilidade de evolução prolongada associadas a elevados graus de incapacidade e de dependência funcional (Cabral, 2013), a par das alterações na estrutura familiar e dos desafios de conciliação entre a vida profissional e os cuidados informais em família (Soeiro et al., 2020; Fronteira et al., 2019), determinaram a criação de respostas intersectoriais ajustadas às necessidades de saúde e sociais.

Na relação intergeracional, são cada vez menos para cuidar de mais, ainda que se mantenha a tendência de responsabilização da família como principal sistema de cuidados e de provisão social informal, sobretudo no âmbito de sociedades familistas típicas dos países do sul da Europa (Guadalupe \& Cardoso, 2018; Portugal, 2014). As famílias são hoje mais pequenas (de 3,1 pessoas para 2,5 pessoas em menos de 30 anos); encontramse repartidas por mais agregados domésticos (que são agora quase mais 1 milhão do que em 1992); há mais pessoas a viver sós (mais 132\% em 27 anos, entre 1992 e 2019), particularmente pessoas com 65 ou mais (os agregados unipessoais cresceram 112\% em 28 anos, entre 1991 e 2019), tendo aumentado 59,0\% os casais sem filhos em 27 anos (entre 1992 e 2019) (Pordata, 2020). A par, verifica-se uma taxa de cobertura de respostas sociais para a população idosa de 12,6\%, com uma taxa de utilização média de 77,0\% para todas as tipologias de respostas, apresentando as Estruturas Residenciais para as Pessoas Idosos uma taxa de ocupação de 93,0\% (Gabinete de Estratégia e Planeamento do Ministério do Trabalho, Solidariedade e Segurança Social, 2019), o que indica uma insuficiência de respostas a nível geral na comunidade e uma notória sobreocupação de respostas que asseguram cuidados permanentes a esta população, particularmente na vertente residencial.

A doença é uma experiência universal ao longo do ciclo de vida, assim como a necessidade de cuidados de terceiros. Se esta necessidade de cuidados é frequentemente transitória, na população mais envelhecida tende a ser mais permanente, dadas as características da senescência e de morbilidade que se associam frequentemente às idades mais avançadas. Estabelece-se uma relação contínua entre os processos de envelhecimento saudável e patológico nos desafios da longevidade, reportando aqui às ideias de 
envelhecimento secundário e terciário, com doença e declínio funcional e cognitivo, respetivamente (Lima, 2010). Note-se que Portugal registou, em 2018, uma média de anos saudáveis após os 65 anos de idade abaixo da média da União Europeia (7,8 vs. 9,8 anos), tendo a Suécia registado precisamente o dobro dos anos de expectativa de vida saudável entre a população idosa, do que Portugal (15,6 anos) (Pordata, 2020).

Emerge, neste contexto demograficamente desafiante, o papel essencial do sistema de saúde, nos seus diferentes níveis de cuidados: primários, hospitalares, continuados e paliativos. Para assegurar o terceiro nível de cuidados, surge a Rede Nacional de Cuidados Continuados Integrados (RNCCl) com o objetivo de promover a continuidade da prestação de cuidados de saúde e de apoio social, em 2006 ${ }^{1}$. Implementa-se um modelo de prestação de cuidados centrados no doente, desenvolvido por dois setores com responsabilidades fundamentais de intervenção para favorecer o bem-estar social em Portugal: o Serviço Nacional de Saúde (SNS) e o Sistema de Segurança Social. Os cuidados continuados integrados (CCl) têm em vista ganhos em saúde, focando a recuperação global da pessoa, promovendo a sua autonomia e aumentando a sua funcionalidade, no âmbito da situação de dependência em que se encontra, com a intenção da sua reintegração sociofamiliar. Esta rede compreende cuidados de natureza preventiva e reabilitadora, constituindo um nível de cuidados de saúde e de apoio social entre o internamento hospitalar e os cuidados de base comunitária. A importância de assegurar a continuidade dos cuidados de saúde a doentes dependentes, bem como assegurar o seu suporte informal ou formal, são pontos fundamentais na preparação de alta hospitalar dos doentes pelo Serviço Social. $O$ assistente social integra, assim, uma equipa multidisciplinar de Gestão de Altas (EGA) ${ }^{2}$ no âmbito da RNCCl e assume um papel central no planeamento da alta a nível hospitalar. A intervenção social que daqui decorre tem por base a qualidade e a adequabilidade à situação problema e ao contexto do cidadão, e é perspetivada holisticamente.

A triangulação entre o doente, a família, a equipa clínica, e as entidades comunitárias, torna-se, assim, central no processo de estudo e planificação da intervenção em Serviço Social entre o planeamento da alta dos cuidados de saúde e o planeamento da continuidade de cuidados na comunidade (Masfret, 2012). Neste contexto, a articulação entre o Serviço Social e o apoio social informal ganha uma especial atenção. Na conceptualização da RNCCl, é salientada a importância da família, a par de outros agentes da sociedade, na garantia dos cuidados. É ainda sublinhada a necessidade de reforço das capacidades e das competências das famílias, assim como o reconhecimento da importância da conciliação das obrigações da vida profissional com o acompanhamento familiar aos doentes ${ }^{3}$.

Ainda que a rede de suporte inclua a família e outros campos relacionais, tais como os amigos, a vizinhança, as relações de trabalho e as relações institucionais com serviços sociais e de saúde, em Portugal a família é "quase sempre tida enquanto principal reduto de suporte em caso de vulnerabilidade, sobretudo na doença", constituindo-se como "núcleo duro da rede primária de suporte social" (Guadalupe, 2012, p. 186). Quando remetemos para a esfera dos cuidados, a família é tida enquanto o centro e o garante dos cuidados aos idosos e dependentes, constituindo os cuidados prestados pelos seus membros, muitas vezes, a única e principal prestação dos cuidados informais necessários (Carval ho, 2015; Pimentel, 2012; Portugal, 2014). Segundo Soeiro et al. (2020, p. 58), a glorificação da família nos cuidados desloca a responsabilidade coletiva e estatal na

\footnotetext{
${ }^{1}$ Cf. Decreto-Lei n.o 101/2006, de 6 de junho.

${ }^{2}$ Cf. Despacho n.o 7968/2011:1,2 e Decreto-lei n.o 101/2006 de 6 de junho, e Portaria 50/2017 de 2 de fevereiro.

${ }^{3}$ Cf. Decreto-Lei n.o 101/2006 que instituiu a RNCCI, e o Decreto-Lei n.o 281/2003, que cria a rede de cuidados continuados de saúde. Neste decreto legislativo refere-se que, além da prestação de cuidados de saúde em geral que a família e a comunidade social têm constituído, devem "continuar a intervir como fatores essenciais e indispensáveis no apoio aos seus concidadãos, nomeadamente aos mais frágeis e carenciados" (Preâmbulo).
} 
prestação de cuidados para as redes informais, funcionando como suas substitutivas. Estima-se que cerca de 12,5\% da população portuguesa esteja envolvida em cuidados informais, isto é, cerca de 1 milhão a 1 milhão e cem mil (Eurocarers, 2020; European Union, 2018), essencialmente assegurados por mulheres (e.g., Soeiro et al., 2020), o que permite uma aproximação à dimensão do universo de cuidados informais.

Apesar de, na sua ação, o Serviço Social hospitalar estabelecer uma estreita articulação com as redes de apoio formal, é com a rede informal que estabelece uma relação direta em primeira instância. Assim, o presente estudo tem por objetivo a análise da articulação com as redes de apoio informal no contexto da continuidade de cuidados a pessoas idosas, com base na análise de dados dos processos sociais de utentes referenciados para RNCCl a partir do hospital. Pretende compreender-se se a continuidade de cuidados de saúde na comunidade depende eminentemente do apoio informal relativamente ao sistema de saúde e às respostas formais.

\section{Método}

\section{Tipo de Estudo}

Este consistiu num estudo descritivo com análise quantitativa univariada, partindo dos registos estatísticos disponíveis acerca dos participantes e da análise categorial de conteúdo dos registos nos processos sociais.

\section{Procedimentos e Questões Éticas na Recolha de Informação}

Na recolha de informação e de análise documental, os procedimentos foram pautados de acordo com princípios éticos e deontológicos ${ }^{4}$. A recolha de dados foi realizada a partir dos processos do Serviço Social hospitalar e a informação social que consta da plataforma SI-RNCCI (aplicativo de monitorização da RNCCI) onde se procede à inscrição e referenciação dos doentes, sendo utilizada pela Equipa de Gestão de Altas (EGA) ${ }^{5}$ hospitalar. Os dados recolhidos na SI-RNCCI estavam previamente categorizados, os restantes dados, que contavam dos registos nos processos sociais do Serviço Social do hospital, foram sujeitos a análise categorial de conteúdo para posterior quantificação. Assim, efetuámos a análise dos processos sociais, a análise dos registos efetuados no processo de continuidade de cuidados e de destino pós-alta, bem como a caraterização do suporte social informal e formal. Estas análises referem-se a três momentos: durante o internamento hospitalar, à data da referenciação para a RNCCl, e no pós-alta de Unidades da RNCCI. O estudo foi aprovado pelo Centro de Investigação e aprovado pela Comissão de Ética do hospital (Processo com Ref. CE n.o 24/20) e pelo Conselho de Administração do Centro Hospitalar (Ata n.o 26 de 15 de julho de 2020).

\footnotetext{
${ }^{4}$ Foi seguido o Código Deontológico dos Assistentes Sociais, reconhecido e adotado pela Associação dos Profissionais de Serviço Social (aprovado na Assembleia geral da APSS em 25 de outubro de 2018), de acordo com o Código de Conduta Ética da DGS, publicado no Aviso n.o 201/2015, do DR II série, n. .5 , de 8 de Janeiro de 2015, bem como de acordo com o Código de Ética do CHL, aprovado em 21-05-2015, através de deliberação do CA, ata $\mathrm{n}$. o 18.

${ }^{5}$ A Equipa de Gestão de Altas (EGA) é uma equipa hospitalar multidisciplinar, sediada em hospital integrado no SNS, que avalia e confirma a proposta de referenciação dos utentes para as unidades e equipas da RNCCI", segundo a Portaria 50/2017, tendo sido instituída pelo Decreto Lei n.o 101/2006 (art.o 12). A EGA é de carácter obrigatório, tal como expresso no Despacho 7968/2011: "em cada hospital do Serviço Nacional de Saúde (SNS) tem de existir uma equipa de gestão de altas". Na sua constituição "integram um médico, um enfermeiro e um assistente social, podendo ainda integrar outros profissionais, nomeadamente para apoio administrativo, sempre que o volume e a complexidade de atividades o justificar", sendo os profissionais que integram a EGA "designados pelo conselho de administração do hospital e exercem as suas funções preferencialmente em regime de tempo inteiro" (Despacho 7968/2011).
} 


\section{Amostragem}

A amostragem foi não probabilística intencional (Ribeiro, 2007). A amostra ficou constituída por doentes sinalizados pela EGA e referenciados para a $\mathrm{RNCCl}$ a partir do movimento assistencial do Serviço de Medicina de um hospital do Serviço Nacional de Saúde, que integra um centro hospitalar da Região Centro de Portugal, no ano civil de 2019. De 923 doentes internados durante o ano de 2019 no serviço atrás mencionado, foram sinalizados 57 doentes para a RNCCl, o que representa $6,2 \%$, da totalidade.

\section{Participantes}

A amostra incluiu 57 doentes. A maior parte dos doentes sinalizados era do sexo feminino, representando $63,2 \%$ da amostra $(n=36)$. As idades variaram entre os 50 e os 93 anos de idade, sendo sobretudo da faixa etária dos idosos de idade avançada $(40,4 \% ; n=23)$, com uma média de 80 anos. A maior parte residia em contexto rural $(64,9 \% ; n=37)$ e quase todos os doentes residiam em habitação própria, à exceção de três doentes que moravam em habitação arrendada e um em habitação cedida por familiar. Relativamente ao estado civil, $56,1 \%(n=32)$ eram casados, seguindo-se os viúvos $(31,6 \% ; n=18)$.

\section{Tabela 1}

Características Sociodemográficas

\begin{tabular}{|c|c|c|c|c|}
\hline & & $N=57$ & $\%$ & Descritivas \\
\hline \multirow{2}{*}{ Sexo } & Feminino & 36 & 63,2 & \multirow{2}{*}{$M o=$ Feminino } \\
\hline & Masculino & 21 & 36,8 & \\
\hline \multirow{4}{*}{ Grupo Etário } & $<65$ anos & 4 & 7,0 & $M=80,14$ \\
\hline & 65-74 anos (idoso jovem) & 9 & 15,8 & $D P=9,206$ \\
\hline & $75-84$ anos (idoso-idoso) & 21 & 36,8 & Mínimo = 50 \\
\hline & $85+$ anos (idoso de idade avançada) & 23 & 40,4 & Máximo = 93 \\
\hline \multirow{2}{*}{ Residência } & Zona Rural & 37 & 64,9 & \multirow{2}{*}{$M o=$ rural } \\
\hline & Zona Urbana & 20 & 35,1 & \\
\hline \multirow{4}{*}{ Estado Civil } & Solteiro & 4 & 7,0 & \multirow{4}{*}{ Mo $=$ casado } \\
\hline & Casado & 32 & 56,1 & \\
\hline & Viúvo & 18 & 31,6 & \\
\hline & Divorciado & 3 & 5,3 & \\
\hline
\end{tabular}

Nota. $N=$ frequência; $M o=$ moda; $M=$ média; $D P=$ desvio-padrão.

\section{Resultados}

Na Tabela 2 apresentamos os doentes referenciados para Unidades ou Equipas da RNCCI pelas diferentes tipologias. Dos 57 doentes referenciados, a maioria $(58 \% ; n=33)$ tinha critérios específicos para admissão em Unidade Média Duração e Reabilitação (UMDR). Os restantes $21,0 \%(n=12)$ apresentavam critérios para Unidade de Convalescença. Para Unidade Longa Duração e Manutenção (ULDM) foram referenciados 12,2\% ( $n$ 
$=7)$, e $8,8 \%(n=5)$ para Equipas de Cuidados Continuados (ECCI). Verificamos que a maioria dos doentes (91,2\%; $n=52)$, apresentava critérios de referenciação para internamento na $\mathrm{RNCCl}$, e $8,8 \%(n=5)$ para manutenção no domicílio com apoio de ECCI.

Dos 57 doentes sinalizados, foram colocados e admitidos nas Unidades de RNCCl 34 doentes (59,7\%). Houve $24,6 \%(n=14)$ de processos cancelados. Desses, metade $(n=7)$, foram cancelados pela EGA devido a fatores como alta clínica, e/ou recusa dos doentes por se sentirem recuperados. A outra metade $(n=7)$ foi cancelada pela Equipa Coordenadora Local da Rede, sobretudo devido a aspetos relacionados com divergências de critérios clínicos de referenciação. A demora média entre a referenciação pela EGA e a admissão nas Unidades foi de 20,6 dias.

No decorrer do processo de referenciação ou a aguardar vaga para admissão na RNCCl, houve 15,7\% de óbitos, tendo-se registado $26,5 \%$ de óbitos durante o internamento em RNCCl, tendo falecido $42,2 \%$ dos sujeitos da amostra.

\section{Tabela 2}

Doentes Referenciados, Admitidos, Cancelamentos e Óbitos por Tipologia da RNCCI

\begin{tabular}{|c|c|c|c|c|}
\hline Tipologias RNCCI & $\begin{array}{c}\text { Referenciados } \\
n(\%)\end{array}$ & $\begin{array}{c}\text { Admitidos } \\
n(\%)\end{array}$ & $\begin{array}{c}\text { Cancelamentos } \\
n(\%)\end{array}$ & $\begin{array}{c}\text { Óbitos } \\
n(\%)\end{array}$ \\
\hline UMDR - Unidade Média Duração e Reabilitação & $33(58)$ & $19(33,3)$ & $7(12,3)$ & $7(12,3)$ \\
\hline UC - Unidade de Convalescença & $12(21)$ & $9(15,8)$ & $3(5,3)$ & $0(0,0)$ \\
\hline ULDM - Unidade Longa Duração e Manutenção & $7(12,2)$ & $3(5,3)$ & $3(5,3$ & $1(1,7)$ \\
\hline $\mathrm{ECCl}$ - Equipa Cuidados Continuados Integrados & $5(8,8)$ & $3(5,3)$ & $1(1,7)$ & $1(1,7)$ \\
\hline Total & $57(100)$ & $34(59,7)$ & $14(24,6)$ & $9(15,7)$ \\
\hline
\end{tabular}

Na Tabela 3 pode verificar-se que 35,0\% $(n=20)$ dos utentes viviam sozinhos (famílias unipessoais). A maior parte $(45,6 \% ; n=26)$ vivia em casal, $7,1 \%(n=7)$ vivia no contexto do que considerámos família cuidadora, isto é, necessitava de cuidados e vivia com os seus cuidadores. Em família nuclear viviam quatro doentes, mantendo-se os filhos no domicílio dos pais. Salientamos que $8,8 \%(n=5)$ dos doentes da presente amostra eram cuidadores de familiares ( 7 dos cônjuges e 1 da mãe) previamente ao episódio de doença que originou o internamento hospitalar.

As entrevistas de avaliação social foram efetuadas com a rede social primária dos doentes, nomeadamente: com filhos $(38,0 \% ; n=8)$, com cônjuge e filhos $(14,1 \% ; n=8)$, só com cônjuge $(7,1 \% ; n=4)$, com amigos/vizinhos (5,3\%; $n=3$ ), e com sobrinhos, com cônjuge e irmãos/cunhados, com filhos e irmãos/cunhados, e só com irmãos (respetivamente, $1,7 \% ; n=1$ ). Sendo os/as filhos/as que representavam o apoio social informal dos doentes da presente amostra, é de referir que a maioria dos doentes tinha filhos/as (91,2\%), 40,4\% tinha dois filhos.

Verificámos ainda que dos doentes com filhos, $42,1 \%(n=24)$ tinham filhos emigrantes, sendo que desses, $19,5 \%(n=11)$ tinham todos os filhos no estrangeiro. Ou seja, do universo dos doentes que tinham filhos emigrantes, em $46,0 \%$ todos os filhos eram emigrantes. 


\section{Tabela 3}

Família, Número de Filhos e Elementos da Rede Social Primária

\begin{tabular}{|c|c|c|c|}
\hline & & $N=57$ & $\%$ \\
\hline \multirow{4}{*}{ Família } & Unipessoal & 20 & 35 \\
\hline & Casal & 26 & 45,6 \\
\hline & Família cuidadora & 7 & 12,3 \\
\hline & Nuclear com filhos adultos & 4 & 7,1 \\
\hline \multirow{6}{*}{ Número de filhos } & Sem filhos & 5 & 8,8 \\
\hline & 1 filho & 13 & 22,8 \\
\hline & 2 filhos & 23 & 40,4 \\
\hline & 3 filhos & 9 & 15,8 \\
\hline & 4 filhos & 6 & 10,5 \\
\hline & + de 4 filhos & 4 & 1,7 \\
\hline \multirow{2}{*}{ Filhos emigrados } & Sem filhos emigrantes & 33 & 57,9 \\
\hline & Com filhos emigrantes & 24 & 42,1 \\
\hline \multirow{8}{*}{$\begin{array}{l}\text { Elementos da } \\
\text { rede social } \\
\text { primária }\end{array}$} & Cônjuge & 4 & 7,1 \\
\hline & Cônjuge e Filhos & 8 & 14,1 \\
\hline & Filhos & 38 & 66,7 \\
\hline & Sobrinhos & 1 & 1,7 \\
\hline & Cônjuge e irmãos/cunhados & 1 & 1,7 \\
\hline & Filhos e irmãos/cunhados & 1 & 1,7 \\
\hline & Amigos/vizinhos & 3 & 5,3 \\
\hline & Irmãos & 1 & 1,7 \\
\hline
\end{tabular}

Como referimos antes, dos 57 doentes referenciados pelo hospital para a rede, registaram-se 14 cancelamentos e nove óbitos antes do ingresso na rede, tendo havido mais nove óbitos durante o internamento em unidades da rede, num total de 32 casos (56,1\%). Dos 25 sujeitos que tiveram alta da RNCCI (43,9\% da amostra), todos apresentavam necessidade de apoio no pós-alta, ao contrário da situação verificada à data da referenciação para a rede, em que apenas $20,0 \%$ tinha apoio, havendo uma diferença significativa entre a distribuição pelos dois momentos $(p=0,001)$. Na presente amostra, a totalidade dos doentes com alta da RNCCI teve necessidade da prestação de cuidados. A maior parte teve apoio informal $(44,0 \% ; n=11), 36,0 \%(n=9)$ teve apoio formal, e $20,0 \%(n=5)$ teve apoio informal e formal. Verificamos, assim, que $64,0 \%(n=16)$ tiveram apoio informal (Tabela 4$)$. 
Note-se que entre doentes que não necessitavam de cuidados, $58,3 \%(n=14)$ tinham filhos emigrados. Os doentes que precisavam da prestação de cuidados, $28,1 \%(n=16)$ era prestada por cuidadores informais, e desses, $75,0 \%(n=12)$, não tinha filhos emigrados.

\section{Tabela 4}

Apoio Formal e Informal à Data da Referenciação e Apoio no Pós-Alta da RNCCI

\begin{tabular}{|c|c|c|c|c|c|}
\hline \multirow[b]{2}{*}{ Avaliação da amostra total } & \multicolumn{2}{|c|}{$\begin{array}{c}\text { Apoio à data da referenciação } \\
\text { para a RNCCl }\end{array}$} & \multicolumn{2}{|c|}{$\begin{array}{c}\text { Apoio no pós-alta da } \\
\text { RNCCl } \\
\end{array}$} & \\
\hline & $n$ & $\%$ & $n$ & $\%$ & \\
\hline Sem necessidade de apoio & 24 & 42,1 & 0 & 0,0 & \multirow{6}{*}{-} \\
\hline Sem apoio & 3 & 5,3 & 0 & 0,0 & \\
\hline Com apoio informal & 16 & 28,1 & 11 & 19,3 & \\
\hline Com apoio formal & 11 & 19,2 & 9 & 15,8 & \\
\hline Com apoio formal e apoio informal & 3 & 5,3 & 5 & 8,8 & \\
\hline Cancelamentos e óbitos & 0 & 0,0 & 32 & 56,1 & \\
\hline Total & 57 & 100 & 57 & 100 & \\
\hline \multicolumn{6}{|l|}{ Avaliação da subamostra com alta } \\
\hline Sem necessidade de apoio & 18 & 72,0 & 0 & 0,0 & \multirow{5}{*}{-} \\
\hline Sem apoio & 2 & 8,0 & 0 & 0,0 & \\
\hline Com apoio informal & 3 & 12,0 & 11 & 44,0 & \\
\hline Com apoio formal & 2 & 8,0 & 9 & 36,0 & \\
\hline Com apoio formal e apoio informal & 0 & 0,0 & 5 & 20,0 & \\
\hline Total & 25 & 100 & 25 & 100 & \\
\hline \multicolumn{6}{|c|}{ Avaliação dicotomizada da subamostra com alta } \\
\hline Sem apoio & 20 & 80,0 & 0 & 0,0 & \multirow{2}{*}{$p<0,001^{a}$} \\
\hline Com apoio & 5 & 20,0 & 25 & 100 & \\
\hline Total & 25 & 100 & 25 & 100 & \\
\hline
\end{tabular}

Nota. ${ }^{\mathrm{a}}$ McNemar Test.

Para aferir da necessidade da prestação dos cuidados a nível das atividades de vida diária aquando da referenciação na $\mathrm{RNCCl}$, recorremos à avaliação de âmbito social efetuada através da Classificação Internacional de Funcionalidade, Incapacidade e Saúde (CIF) (Organização Mundial de Saúde [OMS], 2014), realizada no momento da referenciação pela EGA ${ }^{6}$.

\footnotetext{
${ }^{6} \mathrm{Na} \mathrm{RNCCl}$ esta avaliação é obrigatória para as referenciações de acordo com a Portaria no 50/2017, e pretende acompanhar "desde o momento da referenciação e ao longo de toda a trajetória do utente na Rede" (Preâmbulo). Foram analisados os qualificadores de Atividades e Participação, nomeadamente os referentes à mobilidade: andar ou movimentar-se (d460); lavar-se (d510); cuidar de partes do corpo (d520); vestir-se (d540); comer (d550); e, cuidar da própria saúde (d570).
} 
$\mathrm{Na}$ Tabela 5 verificamos que mais de metade dos 25 utentes avaliados tinha dificuldade completa na maioria dos qualificadores, aquando da referenciação pela EGA. Segundo a avaliação da CIF, representa a percentagem de $96-100 \%$, seguindo-se a dificuldade grave, que representa 50-95\%, e a dificuldade moderada que representa $25-49,0 \%$. Só $12,0 \%(n=3)$ tinha dificuldade ligeira, registando-se no qualificador de comer. Sessenta por cento $(n=15)$ apresentavam dificuldade completa na capacidade de se vestir e mais de metade dos doentes apresentavam dificuldade completa no qualificador da mobilidade (andar ou movimentar-se) ( $n=14 ; 56,0 \%)$ e de cuidar da própria saúde ( $n=14 ; 56,0 \%)$.

\section{Tabela 5}

Qualificadores de Atividade e Participação Avaliados pela Classificação Internacional da Funcionalidade, Incapacidade e Saúde

\begin{tabular}{|c|c|c|c|c|c|c|c|c|c|c|c|c|}
\hline \multirow{2}{*}{ Qualificador/dificuldade } & \multicolumn{2}{|c|}{ D460 } & \multicolumn{2}{|c|}{ D510 } & \multicolumn{2}{|c|}{ D520 } & \multicolumn{2}{|c|}{ D540 } & \multicolumn{2}{|c|}{ D550 } & \multicolumn{2}{|c|}{ D570 } \\
\hline & $n$ & $\%$ & $n$ & $\%$ & $n$ & $\%$ & $n$ & $\%$ & $n$ & $\%$ & $n$ & $\%$ \\
\hline Completa (96 - 100\%) & 14 & 56,0 & 13 & 52,0 & 12 & 48,0 & 15 & 60,0 & 8 & 32,0 & 14 & 56,0 \\
\hline Grave (50 - 95\%) & 8 & 32,0 & 9 & 36,0 & 9 & 36,0 & 5 & 20,0 & 8 & 32,0 & 9 & 36,0 \\
\hline Moderada (25 - 49\%) & 3 & 12,0 & 3 & 12,0 & 4 & 16,0 & 5 & 20,0 & 3 & 12,0 & 2 & 8,0 \\
\hline Ligeira (5 - 24\%) & 0 & 0,0 & 0 & 0,0 & 0 & 0,0 & 0 & 0,0 & 3 & 12,0 & 0 & 0,0 \\
\hline Nenhuma (0 - 4\%) & 0 & 0,0 & 0 & 0,0 & 0 & 0,0 & 0 & 0,0 & 3 & 12,0 & 0 & 0,0 \\
\hline Total & 25 & 100 & 25 & 100 & 25 & 100 & 25 & 100 & 25 & 100 & 25 & 100 \\
\hline
\end{tabular}

Nota. Qualificadores: andar ou movimentar-se (d460); lavar-se (d510); cuidar de partes do corpo (d520); vestir-se (d540); comer (d550); cuidar da própria saúde (d570).

\section{Discussão}

A partir de um estudo de doentes sinalizados e referenciados pelo hospital para a $\mathrm{RNCCl}$, perspetiva-se a articulação entre a saúde e o apoio social informal no âmbito na continuidade de cuidados.

A amostra concentra-se nas idades mais avançadas $(75+$ anos), sendo relevante referir que o índice de envelhecimento no concelho de abrangência do hospital onde o estudo foi realizado é superior ao índice nacional (210,6 idosos por cada 100 jovens no concelho vs. 161,3 a nível nacional - dados de 2019; Pordata, 2020). A nível nacional, a população abrangida pela RNCCl em 2018 tinha idade superior a 65 anos (83,7\%), representando 50,7\% a população com idade superior a 80 anos, sobretudo mulheres $(55,9 \%)$, casados $(46,5 \%)$ ou viúvos $36,9 \%$, o que situa a presente amostra nas características dominantes do movimento assistencial da rede (Administração Central do Sistema de Saúde [ACSS], 2018).

Relativamente à coabitação, a maior parte vive em casal, e $12,3 \%$ no contexto do que denominamos família cuidadora, vivendo com familiares que Ihes prestam cuidados. Na amostra havia 35,0\% doentes que viviam sós, valor superior aos 26,0\% registados no âmbito da RNCCI em 2018 (ACSS, 2018) ou dos 22,5\% de agregados unipessoais a nível nacional em 2019 (Pordata, 2020). 
Salienta-se a importância da família, antes, durante e após o internamento hospitalar, designadamente durante a sinalização e a referenciação, a admissão e o internamento na $\mathrm{RNCCl}$, assim como no pós-alta das unidades da RNCCI. Reconhece-se que são atribuídas às famílias acrescidos níveis de responsabilização nos apoios solicitados aos familiares que carecem de cuidados em todas as fases deste processo. Tal é motivo de preocupação, pois, por outro lado, verifica-se que lhes "são dadas cada vez menos condições concretas para que exerçam efetivamente tais responsabilidades" (Guadalupe \& Cardoso, 2018, p. 226).

Em Portugal têm-se verificado múltiplas alterações sociodemográficas e mutações na estrutura familiar. Todavia, o sistema familiar continua a assumir-se como o centro da solidariedade interpessoal e intergeracional, assegurando o bem-estar e os cuidados necessários dos seus parentes idosos dependentes, como se confirma no presente estudo. A maior parte dos indivíduos da amostra não necessitava de cuidados ou de apoio previamente ao internamento hospitalar; entre os que precisavam de apoio, a maioria tinha apoio informal. Já aquando da alta da RNCCl, 64,0\% têm apoio informal em contexto domiciliário. A nível nacional, 77,0\% do destino pós-alta da RNCCI foi para o domicílio (ACSS, 2018), não havendo, no entanto, informação sobre a continuidade dos apoios necessários a assegurar aos doentes.

Após o internamento em RNCCI não foi possível obter dados concretos, no entanto segundo dados de monitorização da RNCCl, os objetivos da intervenção planeada foram concretizados em 76,0\% dos registos (ACSS, 2018).

No presente estudo a maior percentagem dos doentes tem doenças com sequelas permanentes, associando-se níveis de incapacidade relevantes, que requerem cuidados continuados que não se esgotam na RNCCl. Por isso, considera-se muito importante garantir a avaliação da funcionalidade dos doentes aquando da alta da RNCCl, assim como a avaliação do suporte social disponível e das condições efetivas da rede informal para assegurar esse suporte com qualidade. Os cuidados continuados devem ser, como o nome indica, continuados e não adiados. É sublinhada esta ideia porque o direito a este nível de cuidados não deve cessar com a alta do internamento em unidades da $\mathrm{RNCCl}$, sendo de repensar a ideia da alta em cuidados continuados enquanto cuidados sociais e de saúde na comunidade.

Se antes da doença que originou o internamento hospitalar, a maior parte dos doentes da amostra não necessita da prestação de cuidados, posteriormente, a maior parte apresenta a avaliação de dificuldade completa no desempenho das AVD. Quando a dependência não cessa, não devem também cessar os cuidados continuados, evitando a transferência de responsabilidades no cuidar para sistemas de apoio informal e para os cuidados sociais do formal fora da esfera pública do sistema de saúde.

No presente estudo o apoio informal é prestado, na sua maior parte, pelos filhos dos doentes, o que vai ao encontro de outros estudos (e.g., Alves et al., 2020) e do afirmado por Pimentel (2012, p. 70): “os filhos, e mais especificamente as filhas e as noras, surgem como a principal fonte de apoio aos seus parentes idosos". Tendo verificado que na presente amostra havia uma representatividade de filhos emigrados, o que estabelece barreiras de acesso à rede de suporte intergeracional. Dos doentes com filhos, 42,1\% tinham filhos emigrantes, sendo que $19,5 \%$ tinham todos os filhos no estrangeiro; do universo dos que tinham filhos emigrantes, $46 \%$, todos os filhos eram emigrantes. Face ao contingente de emigrantes portugueses e aos fluxos de saída do país na última década, a emigração coloca desafios concretos no que concerne ao apoio das gerações mais velhas, atendendo à ao papel fulcral dos filhos enquanto fontes de apoio informal.

Um estudo de Pego e Nunes (2018) sobre o cuidado informal de longa duração em Portugal, revela que este se centra na população idosa dependente, sendo assegurada sobretudo por filhos. As autoras chamaram a atenção para uma proporção significativa de idosos dependentes sem qualquer tipo de cuidado informal, apontando a 
insustentabilidade do atual modelo de cuidados baseado no setor informal de cuidados de longo prazo, colocando idosos e cuidadores numa situação crítica e alarmante.

Portugal tem, por seu lado, uma das maiores percentagens de cuidadores corresidentes a nível Europeu a assegurarem cuidado a um membro do agregado familiar (Barbosa et al., 2020), o que torna pouco visível o mundo dos cuidados. Salientamos, por isso, o facto de verificarmos, no presente estudo, que pessoas idosas tendem a cuidar de pessoas idosas, pois $8,8 \%$ dos doentes eram cuidadores de familiares, sendo $7,1 \%$ destes os principais e únicos cuidadores dos seus cônjuges. Esta é uma situação que se verifica frequentemente e com impactes multidimensionais. Cuidar na conjugalidade é uma atividade solitária e com parca retaguarda, como indicam Ornstein et al. (2019). Note-se que 8,8\% da amostra passou de cuidador a pessoa com necessidade de cuidados, com todas as suas implicações.

A articulação com os apoios formais é também muito relevante nestes processos, sobretudo com respostas sociais de centro de dia e de serviço de apoio domiciliário, a representatividade é de 19,3\% no apoio prévio e de $36 \%$ no apoio posterior. A nível nacional, e segundo os dados para a $\mathrm{RNCCl}$, o destino pós-alta das Unidades registado para respostas sociais representou $10 \%$ (ACSS, 2018).

Quando os cuidados são partilhados entre a família e os serviços de apoio social, são obtidos melhores ganhos quer para o doente quer para a família, uma vez que reduz a sobrecarga do cuidador (Petronilho, 2007). Além disso, o cuidado informal intergeracional à geração mais idosa, tem impactes multidimensionais na vida de todos os envolvidos nos cuidados. A cooperação entre o apoio social formal e informal é desejável em quase todas as situações, sendo o Serviço Social um construtor privilegiado de articulações e mediações. No entanto, apenas 5,3\% dos doentes eram apoiados formal e informalmente, simultaneamente no momento de sinalização, passando para 20,0\% posteriormente ao internamento em Unidades de RNCCl. Questionamo-nos sobre as razões desta realidade: serão as respostas sociais existentes desadequadas às necessidades sentidas ou não são acessíveis para a maioria?

Os cuidados continuados assentam numa perspetiva de welfare mix em que se procura responsabilizar agentes públicos e privados, formais e informais (Carvalho, 2012). Trata-se de um modelo simultaneamente encarado como revelador de fracasso da provisão social do Estado, no que concerne à capacidade de tornar possível a realização dos direitos sociais, quando considerada uma responsabilidade do próprio Estado (Pereirinha, 1997). A indefinida política de cuidados em Portugal tem deixado a descoberto uma pluralidade de necessidades dos doentes e dos seus cuidadores (Alves et al., 2020) que colocam em causa a saúde, numa perspetiva holística, e que degradam condições de vida que constituem determinantes da saúde e da doença. Uma das necessidades não cobertas assinaladas por estes autores é financeira. Não pode, assim, ser ignorado no atual estudo, que os encargos relativos a cuidados de saúde são de responsabilidade estatal na RNCCI, mas que o utente suporta, os encargos decorrentes da prestação dos cuidados de apoio social ${ }^{7}$, com comparticipação da segurança social. Ainda que o acesso à rede seja através de prescrição de equipas de um SNS público, universal e tendencialmente

\footnotetext{
${ }^{7}$ Cf. Portaria no 994/2006, art.o 70. Na comparticipação da segurança social são contabilizados os rendimentos do agregado familiar dos doentes (de trabalho, de capitais, prediais, sociais, subsídios, de património mobiliário, imobiliário), não sendo contabilizadas as despesas fixas, que todos os doentes e familiares têm, nomeadamente os encargos com habitação, e outras despesas que alguns têm, nomeadamente pagamento de empréstimos, seguros, etc. Além do valor relativo a encargos com cuidados de apoio social (utente/dia em 2019: UMDR - 20,49 euros; ULDM 31,38 euros - fonte: Anexo da Portaria no 17/2019) a partir de 2013, foi introduzido o encargo com fraldas, a pagar também pelo utente, por dia, em internamentos de ULDM (arto 2, Portaria $\mathrm{n}$-41/2013 - em 2019 era de 1,28 euros /dia). Por outro lado, as entidades promotoras e gestoras, de unidades de internamento de média duração e reabilitação e longa duração e manutenção e de ambulatório ao celebrarem os contratos de prestação de serviços com os utentes e ou seus familiares, poderá ser prevista uma caução, com o objetivo de assegurar o respetivo pagamento do internamento do utente (arto. 11ㅇ, Portaria 343/2015).
} 
gratuito; as características da RNCCl introduzem copagamentos em parte do sistema de cuidados continuados. Para os doentes e familiares, estes encargos financeiros associados à integração nalgumas tipologias da RNCCI, sobrecarregam as despesas do agregado familiar.

Considera-se que constitui potencialmente um obstáculo relevante para o acesso a parte deste nível de cuidados, tal como focado frequentemente pelos familiares nas entrevistas sociais. Este é um motivo de desistência dos processos, no entanto, nem sempre ficam claras as razões de recusa de integração na RNCCl por parte dos doentes ou das famílias. Soeiro et al. (2020) defendem que é fundamental equiparar cuidados sociais a cuidados de saúde, assumindo ambos como responsabilidade de provisão social pública, enquanto medida prioritária de uma nova política de cuidados.

A aprovação recente do Estatuto do Cuidador Informal em Portugal vem regular "os direitos e os deveres do cuidador e da pessoa cuidada" (Lei n.o 100/2019, art.o 1). Apesar de surgir tardiamente, a lei representa um avanço significativo, deixando em aberto vários desafios que se colocam ao suporte social informal futuramente (Fronteira et al., 2019). Considera-se que a RNCCl deveria ter também em consideração a perspetiva e a efetiva participação dos doentes e dos familiares. Incluir a visão dos doentes e familiares é não só uma questão de cidadania, como também a garantia de que os serviços irão corresponder às necessidades reais das pessoas a quem se dirigem, tomando-os como agentes ativos no processo.

A vulnerabilidade social do grupo populacional das pessoas idosas remete-nos para um amplo conjunto de complexos determinantes sociais estruturais, conjunturais e individuais que se conjugam na exposição ao risco social. São disso exemplo, limitações e perda da funcionalidade, perdas relacionais e isolamento social, o corte com o mundo do trabalho e a diminuição da participação social, assim como possíveis quebras financeiras (Guadalupe \& Cardoso, 2018). Tais dimensões não encontram resposta nem se esgotam nos e com os cuidados continuados, na forma como se encontram hoje estruturados.

Apesar de o estudo ter limitações amostrais e nos dados disponíveis, assim como uma delimitação contextual restrita, não permitindo a generalização dos resultados ou das suas implicações, deixa um contributo no sentido da reafirmação da fundamental articulação entre Serviço Social hospitalar e os sistemas de apoio informal. Estreitar mediações é essencial para assegurar a continuidade de cuidados sociais e de saúde na comunidade, havendo que repensar e reforçar as respostas do sistema de saúde e das respostas sociais formais comunitárias. À narrativa de que a RNCCI presta cuidados continuados, deve ser criada uma contranarrativa relativamente à população idosa, que implique todos os intervenientes do setor da saúde e social, mas também as redes primárias e secundárias, salvaguardando a sua heterogeneidade, espelhada pelos cursos de vida e condições socioeconómicas (Guadalupe \& Cardoso, 2018), e as suas necessidades efetivas de continuidade de cuidados continuados de saúde e sociais.

Conflito de interesses | Conflict of interest: nenhum | none.

Fontes de financiamento | Funding sources: nenhuma | none.

Contributos: PM: Recolha de dados; Tratamento de dados; Discussão; Redação do artigo. SG: Orientação da investigação; Discussão; Redação do artigo; Revisões.

Declaração | Acknowledgments: O trabalho resulta de uma Dissertação de Mestrado em Serviço Social apresentada ao Instituto Superior Miguel Torga em 2020, intitulada "Serviço social hospitalar e cuidados continuados: Articulação entre a saúde e o apoio social informal" | The work results from a Master's Dissertation in Social Work presented to Instituto Superior Miguel Torga in 2020, entitled " Hospital Social Work and Continuing Care: Mediation between healthcare and informal social support." 


\section{Referências}

Administração Central do Sistema de Saúde. (2018). Relatório de Monitorização da Rede Nacional de Cuidados Continuados Integrados. SNS. https://www.ipss-acaso.org/site_content/download/7004/779/38

Alves, S., Ribeiro, O., \& Paúl, C. (2020). Unmet needs of informal carers of the oldest old in Portugal. Health and Social Care in the Community, 28, 2408-2417. https://doi.org/10.1111/hsc.13063

Barbosa, F., Voss, G., \& Matos, A. D. (2020). Health impact of providing informal care in Portugal. BMC Geriatrics, 20, Artigo 440. https://doi.org/10.1186/s12877-020-01841-z

Cabral, M. V. (Org.) (2013). Processos de envelhecimento em Portugal. Fundação Francisco Manuel dos Santos. https://bit.ly/2HEZFCS

Carvalho, M. I. (2012). Cuidados continuados integrados e serviço social. Em Carvalho, M. I. (coord.) (Ed.), Serviço social na saúde (pp. 83-110). Pactor.

Carvalho, M. I. (2015). Cuidados familiares prestados a pessoas idosas. Em Carvalho, M. I. (Ed.), Serviço social com familias (pp. 239-250). Pactor.

Eurocarers. (2020). Number of carers and existing support measures across the EU. https://eurocarers.org/aboutcarers/

European Union. (2018). Informal care in Europe exploring formalisation, availability and quality. https://bit.ly/3m7GKzB

Fronteira, I., Simoes, J., \& Augusto, G. (2019). Informal carer in Portugal: Moving towards political recognition in Portugal. European Journal of Public Health, 29(Sup. 4), ckz187.178. https://doi.org/10.1093/eurpub/ckz187.178

Gabinete de Estratégia e Planeamento do Ministério do Trabalho, Solidariedade e Segurança Social. (2019). Carta social - Rede de serviços e equipamentos 2018 [Relatório]. http://www.cartasocial.pt/pdf/csocial2018.pdf

Guadalupe, S. (2012). A intervenção do serviço social na saúde com famílias e em redes de suporte social. Em Carvalho, M. I. (coord.). Serviço social na saúde (pp. 183-217). Pactor.

Guadalupe, S., \& Cardoso, J. (2018). As redes de suporte social informal como fontes de provisão social em Portugal: o caso da população idosa. Revista Sociedade e Estado, 33(1), 215-250. https://doi.org/10.1590/s0102699220183301009

Lima, M. (2010). Envelhecimento(s): Estado da arte. Imprensa da Universidade de Coimbra.

Masfret, D. C. (2012). O serviço social de saúde e o planeamento da alta para a continuidade de cuidados na comunidade. Em Carvalho, M. I. (Ed.), Serviço Social com Famílias (pp. 55-81). Pactor.

Organização Mundial de Saúde. (1999). Glossaire de la promocion de la santé. Organization Mondiale de la Santé. http://www.quebecenforme.org/

Organização Mundial de Saúde. (2014). CIF - Classificação Internacional de Funcionalidade, Incapacidade e Saúde. Direção Geral da Saúde. https://bit.ly/2JccHIB

Ornstein, K. A., Wolff, J. L., Bollens-Lund, E., Rahman, O.-K., \& Kelley, A. S. (2019). Spousal caregivers are caregiving alone in the last years of life, Health Affairs, 38(6), 964-972. https://doi.org/10.1377/hlthaff.2019.00087

Pego, M. A., \& Nunes, C. (2018). Aging, disability, and informal caregivers: A cross-sectional study in Portugal. Frontiers in Medicine, 4, Artigo 255. https://doi.org/10.3389/fmed.2017.00255

Pereirinha, J. (1997). A (re)definição dos direitos sociais face à crise do Estado-Providência e ao fenómeno da exclusão social. Intervenção Social, 15/16, 131-142. https://bit.ly/39c5P8R

Petronilho, F. A. S. (2007). Preparação do regresso a casa. Formação e Saúde.

Pimentel, L. (2012). Cuidar de pessoas idosas dependentes: As interseções entre a esfera pública e a esfera privada. Rediteia, 45, 67-77. https://bit.ly/3mdkC6N

Pordata. (2020). Base de dados de Portugal contemporâneo. Fundação Francisco Manuel dos Santos. https://www.pordata.pt/Home

Portugal, S. (2014). Famílias e redes sociais. Ligações fortes na produção de bem estar. Almedina.

Ribeiro, J. L. P. (2007). Metodologia de investigação em psicologia e em saúde. Legis Editora.

Soeiro, J., Araújo, M., \& Figueiredo, S. (2020). Cuidar de quem cuida - histórias e testemunhos de um trabalho invisível, um manifesto para o futuro. Penguin Random House. 